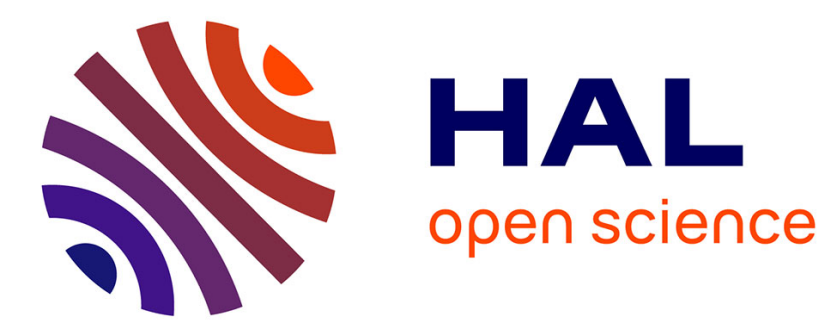

\title{
Une plaque de bronze avec dédicace découverte en Franche-Comté
}

\author{
Gérald Barbet, Robert Billerey
}

\section{To cite this version:}

Gérald Barbet, Robert Billerey. Une plaque de bronze avec dédicace découverte en Franche-Comté. Gallia - Archéologie de la France antique, 2004, 61, pp.281-290. 10.3406/galia.2004.3065 . hal01910548

\section{HAL Id: hal-01910548 \\ https://hal.science/hal-01910548}

Submitted on 19 Jan 2020

HAL is a multi-disciplinary open access archive for the deposit and dissemination of scientific research documents, whether they are published or not. The documents may come from teaching and research institutions in France or abroad, or from public or private research centers.
L'archive ouverte pluridisciplinaire HAL, est destinée au dépôt et à la diffusion de documents scientifiques de niveau recherche, publiés ou non, émanant des établissements d'enseignement et de recherche français ou étrangers, des laboratoires publics ou privés.

\section{(1) (1) $\$$}

Distributed under a Creative Commons Attribution - NonCommercial - NoDerivatives| 4.0 


\title{
UNE PLAQUE DE BRONZE AVEC DÉDICACE DÉCOUVERTE EN FRANCHE-COMTÉ
}

\author{
Gérald BARBET* et Robert BILLEREY**
}

Mots-clés. Romanisation, Gaule, Lingons, épigraphie, évergétisme, cultes, Mars, métallurgie, bronze.

Résumé. En décembre 2000, en bordure de la voie antique reliant Besançon à Pontailler-sur-Saône, sur la localité de Chassey dans la commune de Mutigney (Jura), a été découverte fortuitement une plaque de bronze argenté portant une dédicace gravée. Selon celle-ci, elle accompagnait, à l'origine, une statue de grande valeur.

Le dédicant, un Iulius, prêtre des deux Augustes, l'a offerte à Cicolluis, dieu lingon assimilé à Mars, probablement à la fin du Ir s. Plus tard, la plaque a été enlevée au sanctuaire, brisée et cachée.

Key-words. Romanization, Gaul, Lingones, epigraphy, evergetism, cults, Mars, metallurgy, bronze.

Abstract. In december 2000, a silvered bronze tablet was fortuitously found along the Roman road linking Besanson to Pontaillersur-Saône, at Chassey (commune of Mutigney, Jura). An engraved dedication suggests it was fixed to an upvalued statue.

The dedicant, a Iulius, who is a priest of the two Augusti, offered it to Cicolluis, a god specifically honoured by the Lingones and assimilated to Mars, probably at the end of the $2^{\text {nd }}$ century AD. Later on, the tablet must have been displaced from the temple site, broken and concealed.

Translation : Isabelle FAUDUET

schlagwörter. Romanisierung, Gallien, Lingonen, Epigraphik, Euergetismus, Kulte, Mars, Metallurgie, Bronze.

Jusammenfassung. Im Dezember 2000 wurde auf der Gemarkung von Chassey (commune de Mutigney, dép. Jura) im Randbereich der antiken Straße von Besançon nach Pontailler-sur-Saône zufällig eine versilberte Bronzeplatte mit eingravierter Weiheinschrift gefunden, die ihrem Inhalt nach ursprünglich zu einer sehr wertvollen Statue gehörte.

Der Dedikant, ein Iulius, Priester beider Augusti, hatte sie, wahrscheinlich am Ende des 2. Jahrhunderts n. Chr, dem Cicolluis dargebracht, einer mit Mars gleichgesetzten Gottheit der Lingonen. Später muß die Platte aus dem Heiligtum entfernt, zerbrochen und verborgen worden sein.

Übersetzung: Stefan WIRTH

\section{LA DÉCOUVERTE}

\section{LES CIRCONSTANCES}

Au mois de décembre 2000, en Franche-Comté, dans le département du Jura, a été découverte de façon fortuite une plaque de bronze portant une inscription en latin, dans un fossé bordant la RD 459, sur la commune de Mutigney. L'emplacement de cette découverte est situé à $750 \mathrm{~m}$ de la limite administrative du département de la Côte-d'Or, en région de Bourgogne.

Les précipitations abondantes du mois de décembre ont érodé les parois internes du fossé et permis d'apercevoir une partie de l'objet.

\section{L'ENVIRONNEMENT ARCHÉOLOGIQUE}

La route départementale supplante une voie antique connue. Celle-ci, orientée est-ouest, reliait la Séquanie au territoire des Lingons (fig. 1). Elle traverse les sites de l'agglomération gallo-romaine de Dammartin-Marpain, située en Franche-Comté à $2,5 \mathrm{~km}$ à l'est, et de celle de Pontailler-surSaône, située en Bourgogne à $8 \mathrm{~km}$ à l'ouest. La voie se dirige ensuite vers l'ouest en direction de Mirebeau-sur-Bèze, et vers l'est elle gagne Besançon (Vesontio), capitale de la Séquanie, à 49 km (Mangin et al., 1986 ; Bénard et al. dir., 1994; Rothé, 2001).

Dans ce secteur, les sites occupés à l'époque romaine sont extrêmement nombreux, en bordure de l'Ognon, non loin de

\footnotetext{
* Service régional de l’archéologie de Franche-Comté, 7 rue Charles-Nodier, F-25043 Besançon Cedex.

** Professeur de lettres classiques, 4 rue I apostolest, F-90000 Belfort.
} 


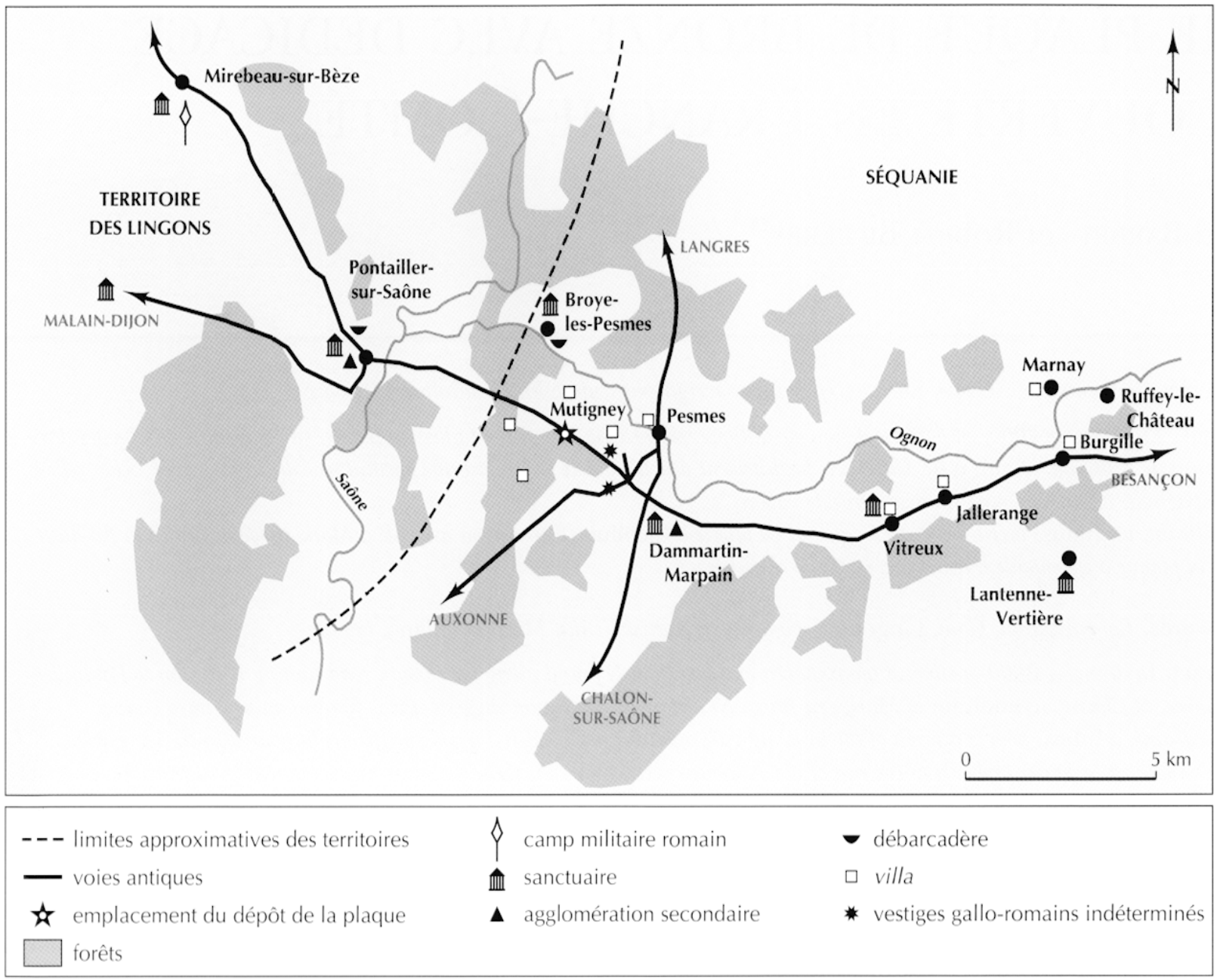

Fig. 1 - Carte de la région de Dammartin-Marpain - Pontailler-sur-Saône : localisation de la découverte (DAO G. Barbet, SRA Franche-Comté).

Tabl. I - Les sites d'époque gallo-romaine autour du confluent de l'Ognon et de la Saône (voir fig. I pour leur localisation).

\begin{tabular}{|c|c|c|}
\hline Localité & Type d'occupation & Références \\
\hline Ruffey-le-Château & groupement rural & SRA Franche-Comté \\
\hline Marnay & importante villa & SRA Franche-Comté \\
\hline Burgille & villa & SRA Franche-Comté \\
\hline Jallerange & villa & SRA Franche-Comté \\
\hline Vitreux & très grande villa & Jaccottey, 1999 \\
\hline Dammartin-Marpain & agglomération secondaire & Mangin et al., 1986 \\
\hline Mutigney & $\begin{array}{l}\text { vestiges indéterminés, } \\
\text { borne milliaire }\end{array}$ & $\begin{array}{l}\text { Mangin et al., } 1986 \\
\text { Rothé, } 2001\end{array}$ \\
\hline Broye-les-Pesmes & $\begin{array}{l}\text { débarcadère sur l'Ognon, } \\
\text { entrepôts (?), } \\
\text { important groupement d'habitats, } \\
\text { sanctuaire }\end{array}$ & $\begin{array}{l}\text { SRA Franche-Comté ; } \\
\text { Dieu, } 1857 \text {; Salsa, } 1987\end{array}$ \\
\hline Pontailler-sur-Saône & $\begin{array}{l}\text { débarcadère sur la Saône, } \\
\text { entrepôts, sanctuaire, } \\
\text { agglomération secondaire }\end{array}$ & Mangin dir., 1994 \\
\hline Mirebeau-sur-Bèze & $\begin{array}{l}\text { sanctuaire, } \\
\text { camp de la VIII légion Auguste }\end{array}$ & $\begin{array}{l}\text { Barbet, } 1981 ; \\
\text { Mangin dir., } 1994\end{array}$ \\
\hline
\end{tabular}

son confluent avec la Saône à $6 \mathrm{~km}$, à Broye-les-Pesmes (tabl. I) (Jaccottey, 1999).

On peut également noter la présence d'une vingtaine de petits sites gallo-romains aux fonctions indéterminées, mais de plus faible importance (Rothé, 2001).

Le village de Chassey, où a été effectuée cette découverte, fait partie de la commune de Mutigney. Les deux agglomérations possèdent sur leur territoire des vestiges gallo-romains indéterminés (SRA Franche-Comté ; Marquiset, 1841, 1842 ; Jaccottey, 1999; Rothé, 2001). Un tronçon de voie antique y rejoint la grande voie Besançon - Pontailler-sur-Saône. Une borne milliaire, aujourd'hui disparue, aurait été découverte au $\mathrm{XIX}^{\mathrm{c}} \mathrm{s}$. à Mutigney. Le terrain labouré jouxtant le lieu de la découverte de la plaque en bronze laisse apparaître quelques rares fragments de tuiles romaines, mais également des tessons de céramique médiévale et moderne.

Les sanctuaires répertoriés sur le tracé de la voie antique sont situés à Lantenne-Vertière (Barbet, Laurent, 1988, 1989, 1995), Vitreux (SRA Franche-Comté), Dammartin-Marpain (Mangin et al., 1986 ; Rothé, 2001), Broye-les-Pesmes (Salsa, 1987 ; Barbet, 2001), Pontailler-sur-Saône et Mirebeau-sur-Bèze (Bénard et al. dir., 1994). 


\section{LA PLAQUE DE BRONZE}

\section{DONNÉES TECHNIQUES}

La plaque, de forme horizontale, mesure $68,2 \mathrm{~cm}$ de longueur et 22,5 à $22,8 \mathrm{~cm}$ de largeur, avec une épaisseur moyenne de $4 \mathrm{~mm}$. Elle pèse $6730 \mathrm{~g}$, ce qui est une masse assez élevée, mais elle est cassée en trois fragments (pour plus de commodité, nous les numéroterons 1, 2, 3, de gauche à droite). La cassure est nette, sans trace de pliure, et ancienne : au bord droit du fragment 2 , une concrétion régulière la suit à environ $1 \mathrm{~cm}$. La fracture s'est faite suivant la ligne de moindre résistance constituée par les jambages des lettres : c'est particulièrement visible entre les fragments 1 et 2 .

Ceux-ci sont approximativement de même taille; le fragment 3 est plus grand, mais un peu plus mince. Tous trois ont été trouvés posés à plat l'un sur l'autre : ils n'ont donc pas ćtć perdus et ne sont pas tombés par hasard. Cette disposition témoigne d'un certain soin pour leur conservation.

Le bronze est de grande qualité : on n'y observe aucune bulle, aucun défaut de fabrication. La patine est gris-vert. La plaque est partiellement recouverte de concrétions très superficielles, surtout côté face et sur le fragment 3 ; à droite, celui-ci est plus fortement oxydé, au point que sa marge est trouée en deux endroits, sans perte de texte.

Cette plaque était fixée, à sa partie supérieure et au verso, par deux attaches en oméga qui ont été conservées (fig. 2) ; chacune était maintenue par deux rivets de fer, maintenant très oxydés, espacés de 36 à $38 \mathrm{~mm}$. Ellc était bordée d'une légère moulure, puis d'un double filet: une même bordure orne une plaque de marbre (CII, VI, 16331: Rome, vers 106) ou un diplôme militaire en bronze (Pannonie, 65) (Rémy, Kayser, 1999, p. 83 et 130). L'ensemble était recouvert d'une feuille d'argent dont il reste une partie sur le fragment 1 , et quelques éléments en bas et au dos des fragments 2 et 3 . Cette feuille d'argent était en effet repliée au verso: le rabat mesure $15 \mathrm{~mm}$ à $17 \mathrm{~mm}$ de largeur et, là où il a disparu, il a souvent laissé une trace nette sur la patine. Il s'agit donc d'une " fausse argenture, par des feuilles d'argent plaquées sur le métal au repoussé : ce travail se fait à froid " (Mangin, 1981, vol. 1, p. 257 n. 1).

Au verso de la plaque apparaissent deux autres séries de traces qui sont sans doute ducs à son mode de fabrication (fig. 3) : d'une part, un alignement longitudinal de petites dépressions oblongues, en amande, sur environ $70 \mathrm{~mm}$ de largeur et, d'autre part, des séries de stries transversales, surtout dans les angles extérieurs et au milieu du fragment 3.

\section{LE TEXTE}

L'inscription, sur quatre lignes, est intégralement conservée. L.es lettres sont élégantes et fermes : c'est une belle quadrata, qui permet de reconstituer un alphabet presque complet (fig. 4). Elles semblent très régulières, sauf un peu à droite où les lignes ont tendance à monter; les derniers mots présentent un graphisme différent du $\mathrm{M}$ et du L, et les I sont plus grands (de telles inégalités de rythme sont « attribuables à la routine et à la lassitude du graveur, [...] plutôt qu'à un changement de

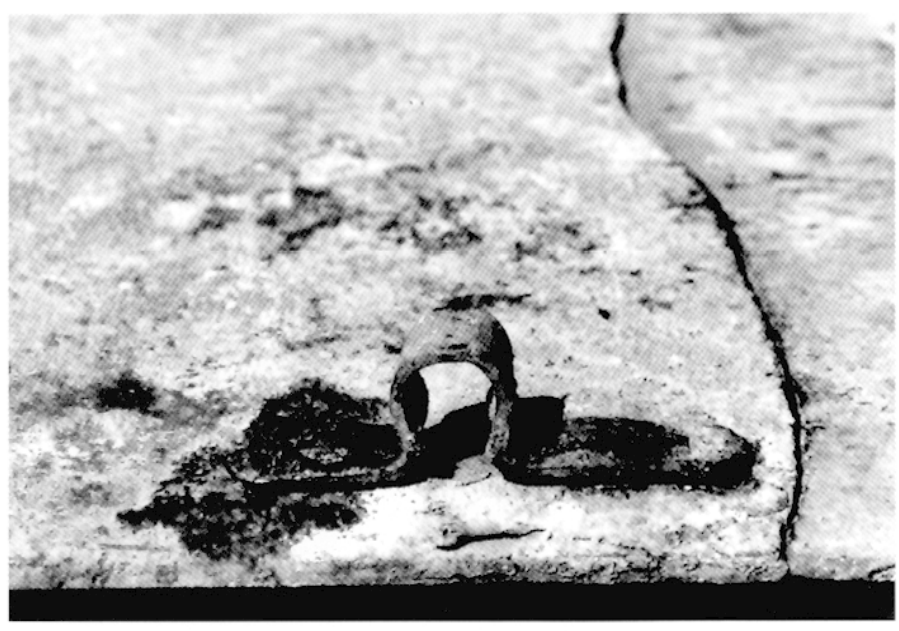

Fig. 2 - Attache en oméga et traces du rabat de la feuille d'argent (cliché R. Billerey).

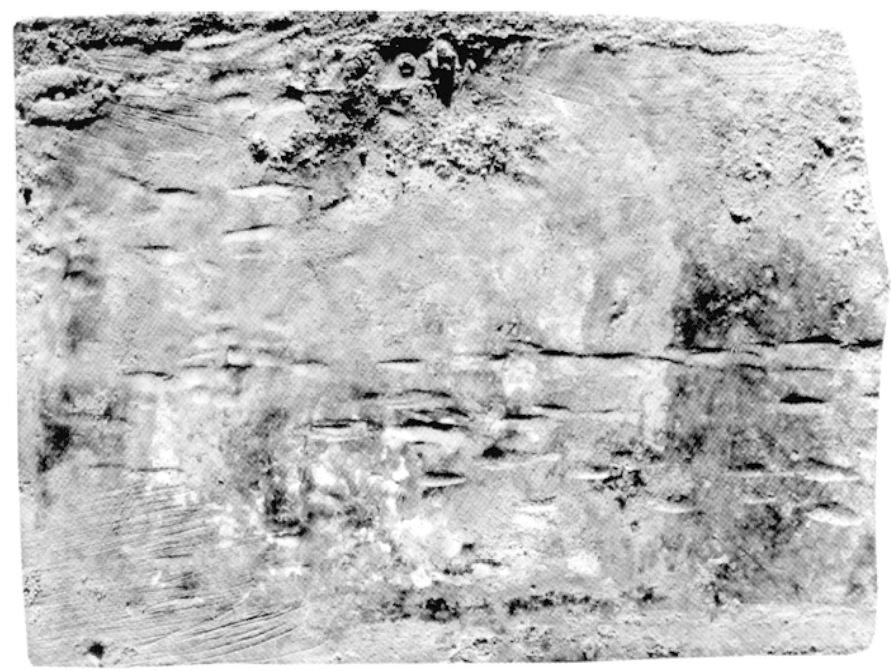

Fig. 3 - Verso de la plaque (cliché R. Billerey).

main ", cf. Duval, Pinault, 1986, p. 22). La dernière ligne est un peu plus haute; ainsi les lettres mesurent entre 25 et $43 \mathrm{~mm}$ de hauteur.

Elles ont été gravées avec une gouge à section triangulaire ; la largeur du tracé atteint parfois $4 \mathrm{~mm}$, surtout dans les hampes verticales (fig. 5). À l'extrémité de celles-ci, on observe souvent un petit bulbe de métal repoussé, visible sur les photographies, qui signifie que la gravure a été effectuée à chaud ou dans une matière ductile (un modèle en cire, cf. Jannet-Vallat, 1990, p. $151 \mathrm{n}^{\circ} 268$ : statuette d'Apollon et Sirona).

Le graveur a d'abord tracé les empattements, puis les hampes, en général de bas en haut et de gauche à droite (Duval, Pinault, 1986, p. 23). Ln trait horizontal unit les lettres $\overline{\mathrm{H}} \overline{\mathrm{S}}$ (sestertium) et surmonte $\overline{\mathrm{N}}$ (nummum) et les lettres numérales $\overline{\mathrm{XXXX}}$ et $\overline{\mathrm{V}} \overline{\mathrm{III}}$. On remarque aussi deux courtes incisions obliques, d'environ $20 \mathrm{~mm}$ de long, dans l'interligne au-dessus de OR de AVGVSTOR(um) et un peu à droite audessus du A de TESTAMENTO. Il s'agit probablement d'apices sur les voyelles $\mathrm{O}$ et $\mathrm{A}$ de ces mots, comme on en observe aussi quelquefois dans l'inscription de la Table Claudienne de Lyon : 

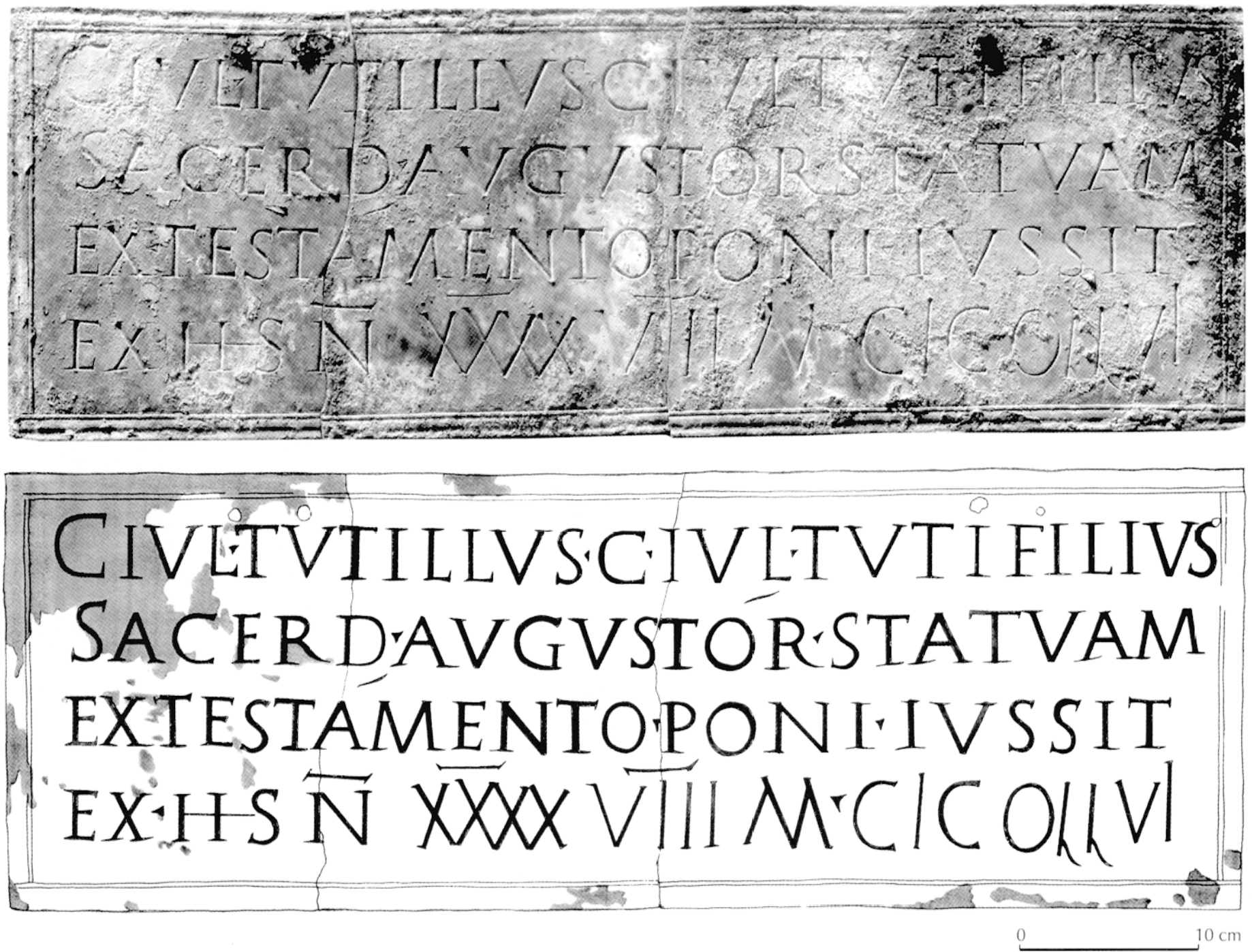

Fig. 4 - Photo et relevé de l'ensemble de l'inscription. Les parties en grisé représentent les traces de la feuille d'argent qui la recouvrait (cliché et dessin R. Billerey).

" accents aigus flexueux qui apparaissent çà et là aux interlignes, posés le plus souvent un peu trop à droite de la lettre qu'ils devraient surmonter ; ces accents désignent toujours des voyelles longues [...], mais ils n'en désignent que quelquesunes parmi beaucoup "; ainsi 1. 46, le A de ADPROBARE, ou 1. 51, sur les O de ORDINIS ORNAMENTVM (Fabia, 1929, p. 56 et photo).

Les mots sont séparés par un point épigraphique triangulaire (onze signes au total): il n'en manque que deux à la première ligne, à moins qu'ils soient dissimulés sous des concrétions.

On lit :

C IVL. TVTILLVS. C. IVL. TVTI FILIVS

SACERD. AVGVSTOR. STATVAM

EX. TESTAMENTO. PONI. IVSSIT

EX. HS $\overline{\mathrm{N}} \overline{\mathrm{XXXX}} \overline{\mathrm{VIII}}$ M. CICOLLVI

C(aius) Iul(ius) Tutillus C(ai) Iul(ii) Tuti filius I sacerd(os) Augustor(um) statuam I ex testamento poni iussit I ex (sestertium) n(ummum) XXXXVIII m(ilibus) Cicollui, ou bien M(arti) Cicollui.

\section{COMMENTAIRE ET ESSAI DE DATATION}

Le personnage se caractérise d'abord par ses tria nomina et ceux de son père. Il s'agit donc au moins d'une deuxième génération romanisée. Si la tribu de rattachement n'est pas mentionnée pour ces citoyens romains, cette absence ne doit pas surprendre : son usage s'estompait jusqu'à disparaître à peu près au III"s. et, " hors de la Narbonnaise, la mention de la tribu ne paraît pas avoir revêtu une grande importance pour les Gaulois romanisés " (Mangard, 1982, p. 42 et notes).

Le cognomen TVTVS et son diminutif TVTILLVS, du type Decmillus Decmani filius (à Til-Châtel en Côte-d'Or, cf. Wuilleumier, 1963, no 412), est manifestement d'origine gauloisc. Les inscriptions donnent en effet de nombreux exemples de cette série onomastique: Tuta, Tuticanus (à Langres), Tuticus, Tuttius... et Tutellus, Toutilla ou Titullus. Mais nous relevons plus particulièrement une Manlia Tuta à Langres (CIL, XIII, 5805), un Valerius Tutus à Eysses (id., 924), un Q. Domitius Tutus à Paris (id., 3183) qui dédic des ex-voto en 

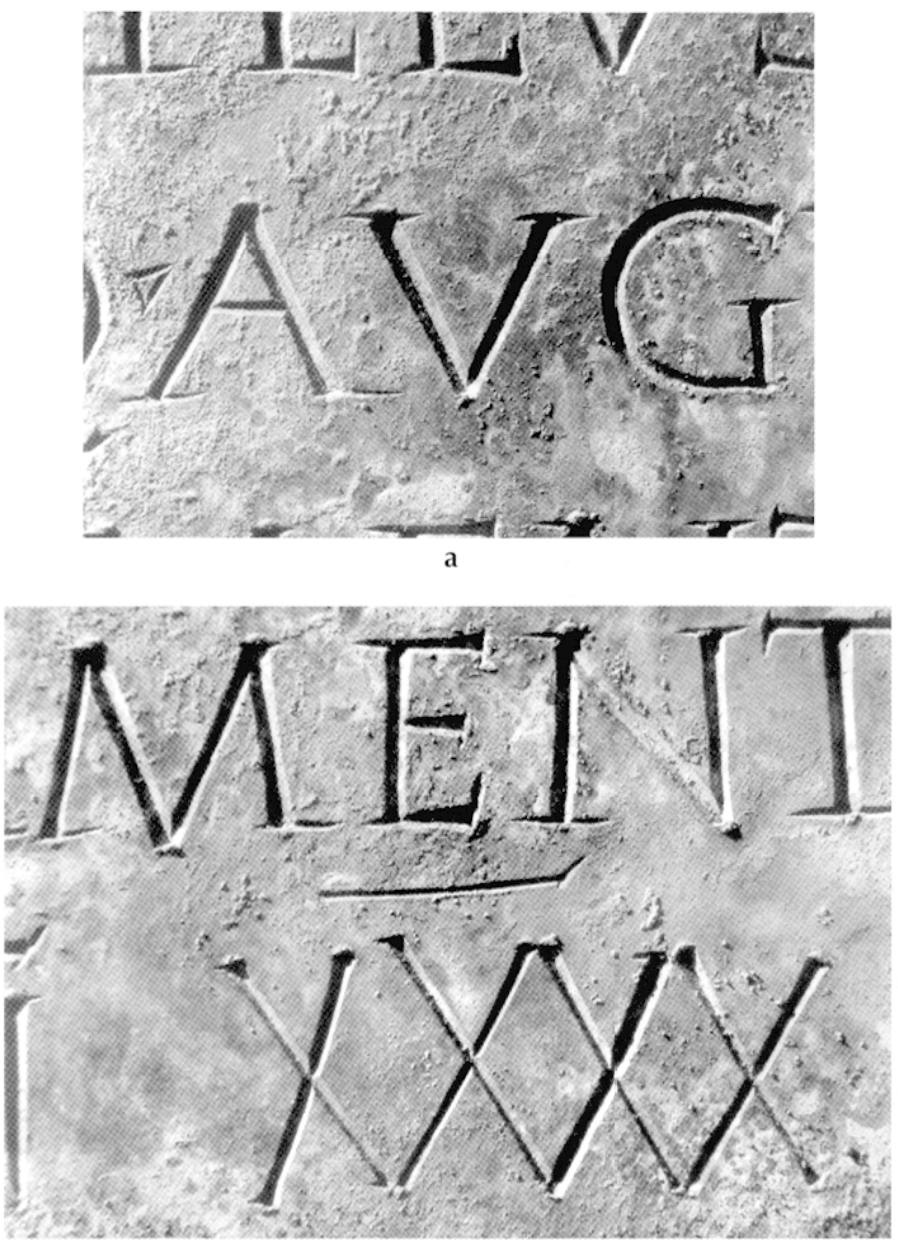

b

Fig. 5 - Vues de détail des lettres (cliché R. Billerey).

céramique. Les marques de potiers citent aussi Tutus ou Tutos: TVTVS F(ecit) à Vichy, TVTI OF(ficina) à Autun, ClermontFerrand, Lyon, et un Tutillus: TOVTILLI M(anu) à Autun et à Marienfels (Allemagne). L'orthographe TOVT- montre qu'un tel nom, assez fréquent, doit être rattaché à la racine teuta-, touta-, " tribu, peuple ", qui a fourni de nombreux dérivés, plutôt qu'à tuto- (Delamarre, 2001, p. 249) : voir Toutius Incitatus, sévir augustal à Lyon et naute de la Saône (CIL, XIII, 1972), et la forme gallo-grecque Toutios, "citoyen ", à Vaison-laRomaine (Lambert, 1994, p. 85).

Signalons encore la stèle de T. Iulius Tuttius, soldat à la XXII Primigenia à Xanten, originaire de Carinthie et mort à Colognte à la fin du $1^{\text {cr }}$ s. (Borger, 1974, p. 84 et 209 ; CIL, XIII, 8289). Ce dernier exemple présente aussi le cas d'un provincial au cognomen indigène, mais pourvu du nomen illustre de Iulius.

Il existe bien d'autres exemples de ce nom, parmi lesquels nous distinguerons, proches de notre C. Iulius Tutus I Tutillus: - (.) Iulius (. filius, sacerdos Romae (et Augusti) ;

- C. Iulius Serenus, sacerdos Romae et Augusti, tous deux à SaintBertrand-de-Comminges (W'uilleumier, 1963, n ${ }^{\circ} 64$ et $74-80$ ) ; - C. Iulius Marinus, fils de C. Iulius Ricoueriugis, flamen Augusti, à Saintes (id., n०149) ;

- C. Tulius C. filius Rufus, sacerdos Romae et Augusti, à Lyon (id., n'217).
La présence de ces Iulii a déjà été observée ailleurs, jusqu'en Afrique ou encore en Orient : C. Iulius Maximus, dont l'épitaphe datée de 155 - 165 a été retrouvée à Philippes (Macédoine), est d'unc famille qui avait reçu la citoyenneté romaine au début de l'Empire, d'Auguste ou de Caligula; tandis que son frère, C. Iulius Teres, a gardé un surnom indigène, et il est thracarches, c'est-à-dire grand-prêtre du culte impérial pour la Thrace (Rémy, Kayser, 1999, p. 110).

L'essor de ces " familles " et leur apparent déclin, qui est plutôt une évolution sociale et économique, ont été fort bien étudiés (Drinkwater, 1978 ; Delaplace, France, 1995, p. 99-102 ; Reddé dir., 1996, p. 150-151). Nous pouvons donc voir en ces Iulii les descendants de la noblesse indigène, dont l'appui au nouveau régime, après la conquête, a été récompensé par l'octroi de la citoyenneté au cours du I ${ }^{\mathrm{er}} \mathrm{s}$. et par une sorte de reconnaissance officielle manifestée par le gentilice qui leur a été conféré.

L.es exemples cités ci-dessus attestent la participation active de ces Iulii au culte impérial. La question de savoir à quel niveau le sacerdos exerçait ses responsabilités a ćté discutée (Mangard, 1982, p. 46) : ce sacerdoce impérial " devait avoir un caractère municipal plutôt que provincial " (Wuilleumier, 1963, p. 26), un caractère « local " (Mangard, 1982); mais il semble qu'entre l'échelon local et le titre fédéral à Lyon existait un niveau intermédiaire seulement provincial (Loriot, Badel, 1993, p. 591 sq. ; Delaplace, France, 1995, p. 74-75). Mais de quelle province ? Dans notre cas, le territoire d'où relève le lieu de la découverte appartenait à la Germanie supérieure depuis Domitien, mais la plaque peut avoir été transportée et provenir du territoire des Lingons, tout proche, qui a été rattaché à la Lyonnaise à une date inconnue, après 150 . Notre personnage n'était pas sacerdos ad aram, ou sacerdos Romae, mais seulement sacerdos Augustorum. En l'absence d'autres rëfërences épigraphiques à son nom, nous devrons considérer qu'il s'agit d'un de ces notables de haut rang, dont l'influence familiale faisait des représentants de leur cité et des interlocuteurs privilégiés de Rome.

Ces personnages investis de fonctions religieuses et politiques pratiquaient souvent l'évergétisme. Si les exemples de l'amphithéâtre de Lyon ou de Saintes sont bien connus, relevons aussi la dédicace du théâtre d'Eu (Seine-Maritime) : L(ucius) Cerialius Rectus, sacerdos R[omae et Aug(usti)] ... Numinibus Aug(ustorum) ... deo [Marti theatru]m ... d(e) s(ua) [p(ecunia) fecit]. (Mangard, 1982, p. 38). L'auteur de cette dédicace, datée des règnes conjoints de Septime Sévère et Caracalla (198-211), a été successivement pruefectus, quaestor, quattuoruir, puis sacerdos, ce qui permet de considérer que cette dernière fonction le place au plus haut rang de sa cité.

Nous retiendrons plus spécialement ici quelques dédicaces de statues :

- à Langres, de Q. Sedulius Sillanus]..., sac(erdos) Aug(usti) arcum s[t]atuas... (CII, XIII, 5688) ;

- à Rennes, de L. Campanius Priscus et de son fils Virilis, sacerdotes Romae et Augusti, statuam cum suis omamentis à leurs propres frais, dédiée à Mars Mullo ;

- à Trèves, de T. Iulius Saturninus, procurator Augustorum au temps de Marc Aurèle et Lucius Verus, une statue monumentale à Asclépios (Schindler, 1970, p. 32 et fig. 87) ; 
- à Lyon, Sex. Iulius Helius accompagne la dédicace d'une statue d'une distribution d'argent aux sévirs augustaux ; et $C$. Satrius, sévir augustal, qui (et) statuam argenteam Libertatis ex libris centum quinquaginta testamento poni iussit, a fait aussi distribuer de l'argent lors de sa dédicace (Wuilleumier, 1963, n ${ }^{\text {os }} 239$ et 240) ; - enfin, à Nantes, Agedouirus et sa fille Toutilla offrent à Augusto Marti Mulloni signum cum suo templo et ornamentis omnibus (CIL, XIII, 3101).

On remarque la similitude des formules et du dernier nom avec la dédicace de Mutigney, et le fait que plusieurs de ces dédicaces sont en l'honneur de Mars. De la même manière, les héritiers testamentaires de C. Iulius Tutillus ne sont pas peu fiers de l'importance de sa donation, au point d'en mentionner le montant. La lecture sestertium nummum pour HS $\bar{N}$ est fréquente, et celle de $\overline{\mathrm{XXXX}} \overline{\mathrm{V}} \overline{\mathrm{II}} \mathrm{M}$ pour 48000 peut être proposée par comparaison avec CII, XIII, 6677, Mayence [dédicace de C. Gentilius Victor : Pro Salute Commodi... ad HS $\bar{N}$ $\overline{V I I I} M I L(i a)$. Faut-il au contraire interpréter le $\mathrm{M}$ de la dernière ligne comme une abréviation de MARTI ? Outre que dans les inscriptions du même genre, ce n'est jamais le cas (voir ci-dessous), le point séparatif après ce $\mathrm{M}$ incite à lire d'un bloc la séquence $\overline{\mathrm{XXXX}} \overline{\mathrm{VIII}} \mathrm{M}$. Un recensement de 135 inscriptions chiffrées concernant des statues (Bang, 1921) relève 13 exemples de chiffres surlignés suivis de $M$. en ce sens, dont CIL, VIII, 14296 : II M. DCXXXXII $\overline{\mathrm{N}}$. qui est sans ambiguïté, 25 exemples où les chiffres sont suivis de MIL. = milia ou milibus et 5 exemples où la mention est encore plus explicite : milib. ou milibus. Il semble donc que la barre de surlignement n'a pas toujours été comprise comme une multiplication par 1000 , mais seulement comme un moyen d'indiquer que les signes surlignés sont des chiffres, par analogie avec $\overline{I I}$ uir $=$ duumuir, $\overline{\text { IIIIII }}$ uir = seuir, par exemple.

Il n'en reste pas moins que la lecture $\mathrm{M}=$ Marti ne peut être éliminée et qu'elle est possible, voire préférable, si l'on retient l'argument que sur huit inscriptions, reprises ci-dessous, qui nomment Cicolluis, sept au moins donnent le double nom Mars Cicolluis. À chacun d'interpréter alors le choix du graveur, qui a pris le soin et la place d'écrire en toutes lettres le nom du dédicant ou le mot testamento et s'est contenté d'un simple $\mathrm{M}$. pour le nom de la divinité.

Le montant de 48000 sesterces est une somme très élevée, si l'on observe que Marc Aurèle et Lucius Verus, coempereurs en 161, offrent alors en donatiuum 20000 sesterces à chaque soldat prétorien (Histoire Auguste, Marc Aurèle, VII, 9), et que la solde annuelle d'un centurion sous Domitien, apparemment inchangée jusqu'à Commode et qui ne sera doublée que sous Septime Sévère en 197, se monte à 18000 sesterces pour un centurion ordinaire, 36000 pour un primus ordo, 72000 pour un primus pilus, un primipile (Speidel, 1996, p. 64-66 et notes), et sur les 135 inscriptions recensées ci-dessus, seules 7 dépassent 48000 sesterces. Le prix des statues se répartit ainsi :

- inférieur à $20000 \mathrm{HS}: 111$, soit $82,2 \%$, dont 22 offertes ex testamento ou par les héritiers ;

- de 20000 à $40000 \mathrm{HS}: 14$, soit 10,4\%, dont 5 ex lestamento; - de 40000 à $60000 \mathrm{HS}: 4$, soit $3 \%$, dont 1 ex testamento;

- puis la valeur fait un bond : supéricur à $100000 \mathrm{HS}: 3$, soit $4,4 \%$, dont 2 ex testamento.
Les statues de valeur proche sont les suivantes :

- pour $40000 \mathrm{HS}$, une statue et un autel (CIL, III, 10305: Dessau, 7126) ; une statue dorée à Rhodiapolis (IGR, III, 739, XIX) ;

- pour $43000 \mathrm{HS}$, une statue de bronze (CIL, XI, 6481) ;

- pour 51335 HS, staluam argenteam à Hippo Regius (CIL, VIII, 17408 : Dessau 5474).

Pour $100000 \mathrm{HS}$ et au-delà, il s'agit souvent de dons collectifs : Gibbenses fecerunt (CIL, VIII, 18547 et 18548) et d'weuvres exceptionnelles: statue équestre, char et statue, quadrige... Notons cependant : simulacrum Viennae [deae] argenteum, pour $200000 \mathrm{HS}$ (CIL, XII, 5864).

Par comparaison avec tous ces exemples, nous pouvons donc penser à une statue de grande taille, ou en métal précieux, assortie à la plaque recouverte d'argent.

Le destinataire de la dédicace, nommé au datif et en caractères plus grands, est le dieu Cicolluis, assimilé au dieu Mars, ou appellation locale de celui-ci (fig. 6). Mars serait cité ici tout au plus par l'abréviation M., par manque de place pour le datif MARTI ou par attachement au seul nom gaulois. Nous le connaissons déjà par quelques inscriptions, la plupart sur des autels de pierre, publiées par Espérandieu (Recueil, passim $\mathrm{n}^{\text {๙ }} 3555$ à 3580 ) et dans le CIL, XIII.

Il y est parfois nommé seul :

- 5479, à Dijon : DEO MARTI CICOLLVI (III"s.) (Drioux, 1934, p. 37$)$;

- 5597, à Mâlain : MARTI . CICOLVI ;

- 5604, à Mâlain : CICO[llui] ?

Une nouvelle dédicace très intéressante est venue s'y ajouter récemment, provenant de Xanten sur le Rhin et datéc de 66-68:

MARTI CICOLLVI SACRVM I PRO SALVTE [(NE I RONIS (AESaris)] AVG(usti)... (Landesmuseum Bonn, voir L'Année épigraphique, 1981, p. 176-177, n. 690 et Jufer, Luginbühl, 2001). Il est aussi invoqué avec Bellone :

- 5598, à Mâlain : maR + CICollui êT BELLonae

ou encore avec LITAVIS :

- 2887, à Aignay-le-Duc, dans la chapelle Saint-Michel : AVGusto . SACrum

DEO MARTI CICOLLVI ET LITAVI ;

- 5599, à Mâlain : MAR $t$ I CICOLLVI . ET LITAVI ;

- 5601, à Mâlain : maR . TI . CIcoLLVI . ET. L.TAVI ;

et probablement les inscriptions fragmentaires :

- 5600, à Mâlain : (Marti Cicollui) ET LITAVI ;

- 5602, à Mâlain, sous la croix du cimetière en 1637: (Marti Cicollui) ET LI'IAVI ;

-5603, à Mâlain : (Marti Cicollui et Lita) Vi uOTVM..

Toutes ces inscriptions ont été trouvées sur le territoire des Lingons, hormis celle d'Aignay-le-Duc, chez leurs voisins les Mandubiens, "peuple secondaire dans la mouvance des Lingons " (Mangin, 1981, vol. 1, p. 334), et la présente inscription, chez les Séquanes, à quelques kilomètres sculement de leur frontière, qui peuvent toutes deux avoir été déplacées; quant à celle de Xanten, les dédicants y sont expressément nommés: ciues lingonum qui... (nom de uicus indéterminé) consistunt: malgré la présence du camp légiommaire de Xanten, il s'agit d'une inscription civile due à des Lingons établis dans le uicus roisin. 


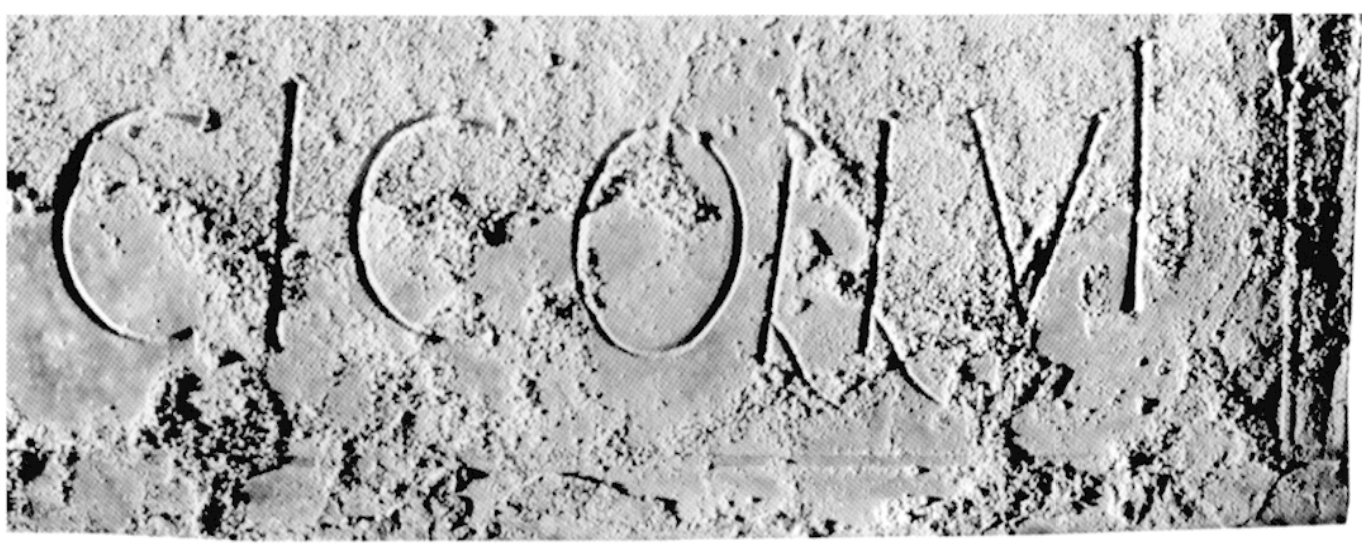

Fig. 6 - Détail inférieur droit : CICOLLVI (cliché R. Billerey).

Le culte de Cicolluis est donc limité à une aire très restreinte dont le centre se situe chez les Lingons, au sanctuaire de Mediolanum/Mâlain. Ce sanctuaire y a été reconnu, à l'ouest d'une grande agglomération gallo-romaine, qui fut un important centre de la métallurgie du bronze. L'ancienne église de Mâlain, dédiée à saint Valérien et démolie vers 1850 , était bâtie à l'emplacement du temple (Drioux, 1934, p. 36 ; Roussel, 1988 ; Jannet-Vallat dir., 1990 ; Bénard et al. dir., 1994). Dans cette dernière référence bibliographique, l'article de L. Roussel, d'après les données des photographies aériennes de R. Goguey, décrit ainsi le sanctuaire: " une grande cour traversée par une conduite d'eau en constitue le cœur, bordée de bâtiments divers dont un fanum carré et une vaste salle basilicale à abside, superposée à une enceinte quadrangulaire à angles arrondis et à des fosses d'époque antérieure ". Sur ce Mars Cicolluis, dieu des Lingons, voir encore la dernière mise au point de W. Van Andringa (2002, p. 145-147).

Bien qu'il soit assimilé à Mars et associé à Bellone, Cicolluis est-il vraiment une divinité guerrière ? Son nom, rapproché du terme cico et interprété en Cic-ollus (Delamarre, 2001, p. 97), signifierait "Gros Muscles ", "le Musclé »: Cicolluis et sa parèdre devraient alors plutôt être considérés comme des dieux nourriciers et protecteurs, et en cela il serait bien conforme aux autres personnifications indigènes du dieu Mars; divinités rurales, ils forment un couple pacifique, peut-être image de la Terre et de l'Eau, apportant la prospérité à la cité (Drioux, 1934). Voir S. Deyts (1987 et 1992, p. 112 sqa.) pour une iconographie commentée de ces divinités protectrices ${ }^{1}$.

1. Observant que l'une de ces dédicaces a ćté rćutilisćc dans une chapelle dédiée à saint Michel archange. comme une autre destinée à Mars Rudianus, É. Thévenot estime que "l'un et l'autre symbolisent les Forces irrésistibles du Bien, qui engagent la lutte contre l'Esprit du Mal, et dont le triomphe renouvelé assure à la fois le maintien de l'ordre divin et le bonheur de l'humanité. " (Thévenot, 1968 , p. 58). Cet auteur signale d'ailleurs que ce Mars Rudianus est souvent associé au cheral, et que le sanctuaire du Magdalensberg, où a été trouvée une grande statue de cheval, était associé à une source sacrée et à un " bâtiment servant de lieu de réunion aux délégués des cités noriques ". Était-ce le même principe à Mâlain ’ Mais la conception d'un dieu combattant pour le Bien n'est assurément pas incompatible avec celle d'un dieu guerrier, qui expliquerait bien l'association avec Bellone, ou sa christianisation en saint Michel ou saint Valérien.
C'est en ce sens de gardien de l'ordre et de protecteur du bonheur de tous que pourrait aussi s'interpréter l'assimilation de ce Mars avec le princeps, dans le culte impérial : il est remarquable que le dédicant soit très souvent sacerdos Augusti. L'association du culte impérial avec une religion locale est connue par de nombreux autres exemples (ainsi à Rennes). Elle s'inscrit dans une politique qui tend à réorienter les cultes traditionnels, y compris au niveau municipal, dans un sens qui les intègre au nouvel ordre romain, et non à les détruire. Sur le fonctionnement local de la religion gallo-romaine, cet exemple illustre donc des analyses auxquelles nous renvoyons (DondinPayre, Raepsaet-Charlier dir., 1999 ; Van Andringa, 2002).

Ainsi, " le culte de Mars indigène s'est développé de façon officielle, dans le cadre d'une propagande politico-religieuse, principalement orchestrée sous les Flaviens par la classe dirigeante. Ce culte semble avoir été pris en charge par le conseil des Trois Gaules. " (Hatt, 1979b, p. 187). Mars est alors invoqué sous diverses appellations: dans une dédicace récemment découverte Num[inibus] Augg[ustorum] deo Marti Camulo, par un Bellovaque nommé Tiberinius Celerianus, installé à Londres, Londiniensium primus, les coempereurs étaient alors probablement Marc Aurèle et Lucius Verus (Current Archaeology, 182, 2002, vol. XVI, 2, p. 48). Il n'est cependant pas prouvé que ces divinités topiques soient préceltiques, selon l'opinion de J.-J. Hatt, et honorées précisément pour faire pièce à l'influence druidique ; de même, les recherches archéologiques n'ont pas encore permis d'établir une relation sûre entre Mars Cicolluis et l'une des représentations en pierre ou en métal qui ont été retrouvées.

La découverte de Mutigney présente tout particulièrement de fortes similitudes avec celles du calendrier de Coligny, dont les fragments ont été trouvés "non loin des restes d'une voie romaine [...] enfouis à environ trente centimètres du sol " " [...] réunis dans une espèce de panier en forme de hotte" (rapport de P. Dissard, 1897-1898, cité in Duval, Pinault, 1986, p. 1 et 3). Si la plaque de Coligny est environ deux fois plus grande que celle-ci, mais comporte un calendrier dont les lettres sont plus petites $(3,5$ à $7 \mathrm{~mm})$ et gravées à froid, la technique de gravure est assez semblable; mais surtout, elle était accompagnée d'une statue de Mars imberbe et nu, de taille naturelle $(1,74 \mathrm{~m})$, dont le type est " connu particulièrement en Gaule et paraît inconnu en Italie ". 
Le calendrier de Coligny est daté de la fin du $\mathrm{II}^{\mathrm{c}} \mathrm{s}$. Pouvonsnous affiner cette datation pour l'inscription de Mutigney? Nous disposons d'éléments institutionnels.

Une datation haute est suggérée par la régularité des lettres, par l'inclinaison des jambages 1 et 4 du $M$ (ils ne deviennent verticaux qu'à la fin du II ${ }^{\mathrm{e}}$ s., cf. Duval, Pinault, 1986, p. 26) et par la présence des tria nomina (ils se généralisent à la fin de l'époque julio-claudienne ; après 150 , le praenomen a tendance à disparaître, cf. Rémy, Kayser, 1999, p. 79-80, mais les tria nomina persistent plus longtemps chez les notables, cf. Dondin-Payre, Raepsaet-Charlier dir., 2001), et le nom des Iulii renvoie aux débuts de l'Empire ; le nom de la divinité invoquée n'est généralement pas précédé du terme deo avant la fin du $\mathrm{II}^{\mathrm{C}} \mathrm{s}$. (Mangard, 1982, p. 40, n. 10 et 11). Inversement, les empattements horizontaux n'apparaissent guère qu'après 170 (Duval, Pinault, 1986, p. 26), et surtout la mention du sacerdos Augustorum permet de définir la référence historique: les seules périodes, en datation haute, où soit attesté le règne conjoint de deux empereurs, sont en effet :

161-169 : Marc Aurèle et Lucius Verus ;

177-180 : Marc Aurèle et Commode ;

198-209 : Septime Sévère et Caracalla...

La première période possible est donc celle de Marc Aurèle. Or, c'est aussi sous le règne de cet empereur que l'on signale, en 172, des " troubles chez les Séquanes " (res etiam in Sequanis turbatas, in Histoire Auguste, Marc Aurèle, XXII, 10), sans qu'il s'agisse d'une véritable rébellion impliquant une campagne militaire, et la Porte Noire, arc de triomphe érigé à l'entrée de la cité de Vesontio (Besançon), capitale des Séquanes, date probablement de 175, après la "guerre germanique " contre les Marcomans (Walter, Bruchet, 1986, vol. 1, p. 369-379). En l'absence d'indications qui justifieraient une affirmation plus précise, nous pourrons donc retenir le dernier tiers du II"s.

Quant aux circonstances et à la date de l'enlèvement et de la dissimulation de la plaque, nous n'en connaissons rien. Mais les remarques faites à propos du calendrier de Coligny nous semblent tout à fait appropriées ici : la plaque du calendrier et la statue "n'ont pas été brisées en centaines de morceaux pour être refondues mais pour être détruites; une partie des fragments, au moins, a été sauvée de l'anéantissement dans une cachette." (Duval, Pinault, p. 37). Nous dirions plutôt qu'elles ont été détruites pour être mises à l'abri. Ce " pieux sauvetage " serait-il aussi le cas pour la plaque de Mutigney? Et nous garderons la même conclusion : " il n'est pas obligatoire que le sanctuaire supposé ait été situé sur l'emplacement de Coligny (Mutigney) [...]. Les fragments [...] ont pu être apportés d'ailleurs, d'un lieu de culte situé à plusieurs kilomètres de là. "

$$
\text { * * }
$$

La dédicace découverte en décembre 2000 en bordure de la voie antique reliant Besançon à Pontailler-sur-Saône, sur le territoire de la commune de Mutigney, indique qu'elle accompagnait, à l'origine, une statue de grande valeur. Le dédicant, fier de son nom qui le place parmi les Iulii, mais dont le surnom est d'origine indigène, est un grand-prêtre du culte impérial. La référence aux deux Augustes incite à dater cette dédicace de la fin du II $^{\mathrm{c}} \mathrm{s}$.

Celle-ci, seule subsistante, a visiblement été transportée, et le sanctuaire d'origine ne peut être déterminé. L'importance de ce don et le rang élevé du dédicant font exclure l'hypothèse d'une petite chapelle de campagne, mais il n'existe pas d'indices d'un grand sanctuaire à proximité - même si l'on ne doit pas négliger des sites comme Mirebeau-sur-Bèze, malgré la disparition du camp militaire à cette époque (Bénard $e t a l$. dir., 1994), pas plus que les agglomérations de Dammartin-Marpain et de Pontailler-sur-Sâone. Quant à Vesontio, aucun élément ne permet à ce jour d'y envisager l'existence d'un culte à Cicolluis.

Le seul lieu de culte actuellement connu est le sanctuaire de Mâlain, et Cicolluis est identifié comme un dieu spécifiquement lingon. La présente inscription, qui est la neuvième à le mentionner et qui a été trouvée à proximité immédiate de la route qui menait à Dijon et à Mâlain, ne s'oppose pas à cette identification, sans que nous puissions toutefois l'affirmer avec certitude. À une époque indéterminée, la plaque a pu être enlevée à son sanctuaire, brisée et soigneusement mise en dépôt ou cachée. La comparaison avec le calendrier de Coligny, qui paraît avoir connu les mêmes conditions, suggère que l'on avait ainsi l'intention de la préserver plutôt que d'en réutiliser le métal. Ce sera désormais chose faite, grâce aux bons soins du musée de Lons-le-Saunier. 


\section{BIBLIOGRAPHIE}

\section{BANG M.}

1921 : "Preise von Statuen ", in Wissowa G. (DIR.), Darstellungen aus der Sittengeschichte Roms in der Zeit von August bis zum Ausgang der Antonine von Ludwig Friedländer, t. IV, Leipzig, Hirzel, p. 312-325.

\section{BARBET G.}

1981 : Contribution à l'étude de la VIII légion romaine, Besançon, Société de Numismatique du Doubs, $42 \mathrm{p}$.

2001 : Rapport relatif à la découverte d'un sanctuaire gallo-romain sur la commune de Broyeles-Pesmes, Besançon, Service régional de l'archéologie de Franchc-Comté, 21 p.

Barbet G., Laurent H.

1988 : Le sanctuaire gallo-romain de LantenneVertière, Rapport de fouilles, Besançon, Service régional de larchéologic de Franche-Comté.

1989 : Ie sanctuaire gallo-romain de LantenneVertière, Rapport de fouilles, Besançon, Service régional de l'archéologie de Franche-Comté

1995 : " Le sanctuaire gallo-romain de Lantenne-Vertière ", in Eclats d'histoire, 10 ans d'archéologie en Franche-Comté, Besançon, Cêtre, p. 279-283.

Bénaro J., Mangin M., Goguey R., Roussel L. (DIR.)

1994 : Les agglomérations antiques de Cote-d'Or; Paris, I.es Belles I ettres (coll. Annales littéraires de l'université de Besançon, 522, série archéologie, 39), 303 p., 79 fig., $28 \mathrm{pl}$.

BORGER H. (DIR.)

1974 : Römisch-Germanisches Museum, Köln, Verwaltung der Museen der Stadt Köln (coll. Kölner Römer-Illustrierte, 1), 272 p.

Cagnat R.

1898: Cours d'épigraphie latine, Paris, A. Fontemoing, $469 \mathrm{p}$.

DelamarRe $X$.

2001 : Dictionnaire de la langue gauloise, Paris, éd. Frrance, $352 \mathrm{p}$.

Delaplace C., France J.

1995 : Histoire des Gaules (IT' s. av. J.-C./IT s. apr. J.-C.), Paris, Armand Colin, $189 \mathrm{p}$ DEYTS $\mathbf{S}$.

1987 : Divinités indigènes en Bourgogne à l'époque gallo-romaine, Catalogue d'exposition, Dijon, musée archéologique, 46 p., 10 pl.

1992: Images des dieux de la Gaule, Paris, éd. Errance, $159 \mathrm{p}$.

DiEu $\mathrm{H}$.

1857 : Atlas cantonal de la Haute-Saône, Carte du canton de Pesmes.

DONDIN-PAYRe M.

1999 : « Magistratures et administration municipale dans les Trois Gaules ", in DoNolN-
PAYRE M., RAEPSAET-Chari.IER M.-T. (DIR.), Cittes, municipes, colonies. Les processus de municipalisation en Gaule et en Germanie sous le Haut-kmpire romain, Paris, Publications de la Sorbonne, p. 127-230.

Dondin-Payre M., RaePsaet-Charlier M.-T. (DIR.)

1999 : Cilés, municipes, colonies. Les processus de municipalisation en Gaule et en Germanie sous le Haut-Empire romain, Paris, Publications de la Sorbonne, $483 \mathrm{p}$.

2001 : Noms, identités culturelles et romanisation sous le Haut-Empire, Bruxelles, I. I.ivre Timperman, $774 \mathrm{p}$.

DrinkWATER J. F.

1978: "The Rise and Fall of the Gallic Iulii : Aspects of the Development of the Aristocracy of the Three Gauls under the Early Empire ", Latomus, XXXVII, 4, p. $817-850$.

Drioux G.

1934 : Cultes indigènes des Lingons, Paris, Picard, $227 \mathrm{p}$.

Duval P.-M., Pinault G.

1986: Recueil des inscriptions gauloises (RIG) -IIILes calendriers (Coligny; Villards-d'Héria), Paris, éd. du CNRS (coll. Suppl. à Gallia, XIV), 442 p., 75 fig., 6 dépliants.

FABIA $P$.

1929 : La Table Claudienne de Lyon, I.yon, M. Audin, $167 \mathrm{p}$.

HaTt J.J.

1979a: "Le culte de Mars indigène dans le nord-est de la Gaule (sur les pas d'Émile Thévenot) ", Revue archéologique de l'Est, XXX, 1-2, p. 121-138.

1979b: " Le culte de Mars indigène dans le nord-est de la Gaule (sur les pas d'Émile Thévenot) ", Rerue archéologique de l'Est, XXX, 3-4, p. 183-196.

1985 : "Apollon guérisseur en Gaule ", in Peilittifr A. (Dir.), La médecine en Gaule, Paris, Picard, p. 205-238.

\section{JACCOTTEY L.}

1999 : Rapport de prospection systématique de la basse vallee de l'Ognon, Service de la carte archéologique, Service régional de l'archéologie de Franche-Comté, Besançon.

\section{JANNET-VALLAT M. (DIR.)}

1990: Il était une fois la Côte-d'Or: 20 ans de recherche archéologique, Musée archéologique de Dijon, Paris, éd. Errance, $248 \mathrm{p}$.

JUFER N., LUGINBÜhL T.

2001 : Répertoire des dieux gaulois. Les noms de divinités celliques connus par l'éprigraphie, les textes antiques et la toponymie, Paris, éd. Errance, $132 \mathrm{p}$.

\section{LAMBERT P.-Y.}

1994 : La langue gauloise, Paris, éd. Frrance, $239 \mathrm{p}$.

LASSERE J.-M.

1977 : Vbique populus. Peuplement et mouvements de population dans l'Afrique romaine de la chute de Carthage à la fin de la dynastie des Sévères, Paris, éd. du CNRS (coll. Études d'Antiquités africaines), 716 p., 69 ill., 12 cartes.

\section{LoRIOT X., BADEL C. (DIR.)}

1993 : Sources d'histoire romaine $\left(r^{\prime}\right.$ s. av. J.-C./ se s. apr. J.-C.), Paris, Larousse, $858 \mathrm{p}$.

\section{MANGARD M.}

1982 : "L'inscription dédicatoire du théâtre du Bois l'Abbe à Eu (Seine-Maritime) ", Gallia, 40, 1, p. 35-51.

\section{MANGIN M.}

1981: Un quartier de commersants et d'artisans d'Alésia, Scmur-en-Auxois, Lniv. de Dijon/SCHN, 2 vol. : 399 p. ct 301 p.

Mangin M., JacQuet B., JaCob J.-B.

1986: Les agglomérations secondaires en FrancheComté romaine, Paris, Les Belles Lettres (coll. Annales littéraires de l'université de Besançon, 337, série archéologie, 34), 257 p., 2 dépliants.

\section{MARQUISET A.}

1841 : Statistique historique de l'arrondissement de Dôle, vol. 1, Besançon, Charles Deis, 518 p., 15 ill.

1842 : Statistique historique de l'arrondissement de Dôle, vol. 2, Besançon, Charles Deis, 535 p., 12 ill.

\section{RAEPSAET-ChaRLIER M.T.}

1999 : "Les institutions municipales dans les Germanies sous le Haut-Empire ", in DONDIN-PAYRE. M., RAEPSAET-Chari.IFR M.-T. (DIR.), Cités, municipes, colonies. Les processus de municipalisation en Gaule et en Germanie sous le Haul-kmpire romain, Paris, Publications de la Sorbonne, p. 271-352.

REDDÉ M. (DIR.)

1996 : L'armée romaine en Gaule, Paris, éd. Errance, $278 \mathrm{p}$.

RÉmY B., KAYSER F.

1999 : Initiation à l'épigraphie grecque et latine, Paris, éd. Ellipses, 192 p.

\section{ROTHÉ M.-P.}

2001 : Le Jura, 39, Paris, Académie des inscriptions et belles-lettres (coll. Carte archeo logique de la (aaule), 839 p., 553 ill.

\section{Roussel L.}

1988 : Mediolanum. Une bourgade romaine, Dijon, musée archéologique, $324 \mathrm{p}$.

SCHEID J.

1999 : "Aspects religieux de la municipalisa- 
tion ", in DONDIN-PAYre. M., RAFPSAFTCharlater M.-T. (DIR.), Cités, municipes, colonies. Les processus de municipalisation en Gaule et en Germanie sous le Haut-Empire romain, Paris, Publication de la Sorbonne, p. 381-423.

SCHINDLER R. vON

1970 : Landesmuseum Trier, Trier (Trèves), Selbstverlag des Rheinischen Landesmuseums, $87 \mathrm{p}$.

SociétÉ d'AGRICUlture, LetTres, Sciences et arts de la Haute-Saóne (SAlSa)

1987 : Dictionnaire des communes de la HauleSaône, II, Vesoul, Salsa, p. 36-40.

SPEIDEL M. A.

1996 : Die Römischen Schreibtafeln von
Vindonissa, Brugg, Gesellschaft ProVindonissa, $271 \mathrm{p}$.

\section{THĖVENOT É.}

1968 : Divinités et sanctuaires de la (jaule, Paris, Fayard, $245 \mathrm{p}$.

\section{VAN ANDRINGA W.}

1999 : "Prêtrises et cités dans les Trois Gaules et les Germanies du Haut-Empire ", in DONDIN-PAyre: M., RAFPSAFTCharliter M.-T. (DIR.), Cilés, municipes, colonies. Les processus de municipalisation en Gaule et en Germanie sous le Haul-kmpire romain, Paris, Publications de la Sorbonne, p. 425-446.

2002 : I.a religion en Gaule romaine, Paris, éd. Errance, $335 \mathrm{p}$.
WALTER H., BRUCHET J.

1986 : La Porte Noire de Besanson : contribution a l'étude de l'art triomphal des Gaules, 2 vol., Paris, Les Belles Lettres (coll. Annales littéraires de l'université de Besançon, Centre de recherches d'histoire ancienne), $485 \mathrm{p}$.

WISSOWA G., FrIEDLÄNDER L. (DIR.)

1921 : Darstellungen aus der Sittengeschichte Roms in der Zeit von August bis zum Ausgang der Antonine von Ludwig Friedländer; IV, Leipzig, Hirzcl, 336 p.

WUILLEUMIER P.

1963 : Inscriptions latines des Trois Gaules (France), Paris, éd. du CNRS (coll. Suppl. à Gallia, XVII), $256 \mathrm{p}$. 